\title{
Efeito do pH na cinética das reaçōes de oxigênio e monóxido de carbono com hemoglobina de Loricariichthys - peixe aeróbico [*]
}

\author{
A. Focesi Jr. ('); M. Brunori ( ${ }^{2}$ ); J. Bonaventura ( $\left.{ }^{3}\right) ;$ M. T. Wilson ( $\left.{ }^{4}\right) ;$ M. I. Galdames-Portus ( $\left.{ }^{5}\right)$
}

\begin{abstract}
Resumo
Loricariichthys sp., um peixe aeróbico do rio Amazonas, tem um componente hemoglobínico principal. Estudos quantitativos na cinética de dissociação de $\mathrm{O}_{2}$ e combinação de $\mathrm{CO}$ a proteína foram efetuados por experimentos "stopped-flow" a diferentes valores de $\mathrm{pH}$ e uma concentração constante de $1,25 \mathrm{mM}$ de ATP. A dissociação de oxigênio mostra um comportamento simples de $1 .^{\text {* }}$ ordem e uma forte dependência de $\mathrm{pH}$. Por outro lado, a cinética da combinação de $\mathrm{CO}$ foi homogênea e rápida a valores altos de $\mathrm{pH}$, e lenta e claramente autocata lítica a valores de $\mathrm{pH}$ abaixo de 7,0 .
\end{abstract}

\section{INTRODUÇÃo}

As adaptações anatômica e fisiológica dos peixes aeróbicos da família Loricariidae permite-os viver sob variadas concentrações de oxigênio. Espera-se que alguns dos mecanismos de adaptação erivolvam as hemoglobinas, pois as necessidades de oxigênio das espécies de Loricariichthys são maiores do que para outros peixes. (Britsky, E., comunicação pessoal). Para alcançar uma correlação entre as demandas respiratórias e as propriedades funcionais da hemoglobina, sãc necessários estudos qualitativos. Neste artigo, publicamos alguns resultados sobre a cinética das reações de $\mathrm{O}_{2}$ e CO da hemoglobina de Loricariichthys em função do $\mathrm{pH}$. Observe-se que o hemolisado de Loricariichthys contém uma banda principal de homoglobina encontrada por eletroforese em gel de poliacrilamida a qual aparece difusa e pode esta ser quimicamente heterogênea. A relação entre estes espécimes e os descri- tos por Fyhn et al., (1978) é incerta. Como Fink \& Fink enfatiza, a classificação dos Loricariidae está num estágio primitivo. $\mathrm{Na}$ verdade, um espécime de Loricariichthys sp. revelou ser de uma espécie ainda não previamente descrita (Fyhn et al., 1978; Fink \& Fink, 1978). Os resultados publicados, embora preliminarmente, são mais do que nunca úteis como passo inicial para o entendimento das propriedades funcionais completas do sangue de Loricariichthys.

\section{MATERIAL E MÉTODOS}

O hemolisado foi preparado por lavagem dos glóbulos vermelhos recém-extraídos com $\mathrm{NaCl}$ a $1,7 \%$, EDTA $10^{-3} \mathrm{M}$ e pH 7,5. A hemolise foi efetuada por suspensão dos glóbulos vermelhos em 1-2 volumes de EDTA $10^{-3} \mathrm{M}$,

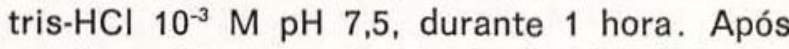
a adição de uma solução de $\mathrm{NaCl} 1, \mathrm{OM}$ para uma concentração final de $0,1 \mathrm{M}$, o hemolisado foi deixado por duas horas e centrifugado a $15.000 \mathrm{rpm}$ (Sorvall RC-2B) para remover fragmentos celulares. Os experimentos cinéticos foram conduzidos em um aparelho "stoppedflow" Durrum-Gibson (Gibson \& Milves, 1964). A concentração de hemoglobina foi medida usando-se um coeficiente de extinção milimolar de $E=13,8$ a $540 \mathrm{~nm}$ na forma oxi (Antonini \& Brunori, 1971). A concentração de monóxido de carbono foi calculada por titulação de miơglobina de cachalote com a solução ga. sosa unida ao ligante.

$(\bullet)$ - Versão original inglesa publicada em Comp. Biochem. Physiol. vol. 62 A (1). 1979.

(1) - Departamento de Bioquímica, I.B., Universidade Estadual de Campinas 13100 Campinas, SP. Brasil.

(2) - CNR Centre for Molecular Biology, Institutes of Chemistry and Biochemistry, Faculty of Medicine, University

(3) - Duke University Marine Laboratory, Beaufirt, N.C. 285516, USA. Established Investigator of the American Heart Association.

(4) - Department of Chemistry, University of Essex, Wivenhoe Park, Colchester $\mathrm{CO}_{4} 3 \mathrm{SQ}$, England.

( 5 ) - Instituto Nacional de Pesquisas da Amazônia, Caixa Postal 478, 69000 Manaus, AM. Brazil. 


\section{RESUltados E Discussäo}

\section{a) CINÉTICA DA DISSOCIAÇÃO DE OXIGÊNIO}

A constante de velocidade de $1^{\mathrm{a}}$ ordem para a dissociação de oxigênio a diferentes valores de $\mathrm{pH}$ foi obtida misturando-se rapidamente: (i) uma solução de hemoglobina equilibrada com ar em $2 \mathrm{mM}$ de tris $\mathrm{pH} 8,0$ e 2,5 $\mathrm{mM}$ de ATP com (ii) tampões sem gás (a diferentes valores de $\mathrm{pH}$ ) contendo uma concentração de cerca de $1 \mathrm{mg} / \mathrm{ml}$ de $\mathrm{Na}_{2} \mathrm{~S}_{2} \mathrm{O}_{4}$. A dissociação de oxigênio segue um comportamento simples de $1^{\text {a }}$ ordem. A homogeneidade do curso de tempo desta reação sugere que a homogeneidade eletroforética não está associada com a heterogeneidade funcional grosseira.

A Fig. 1. descreve a dependência de pH na constante de velocidade de $1^{a}$ ordem para a dissociação de $\mathrm{O}_{2}$, e mostra um marcado efeito Bohr normal. A linha contínua é uma curva de titulação teórica para a dependência de $\mathrm{pH}$ de $\mathrm{K}$, com os parâmetros característicos relacionados na Tabela I. Advirta-se que, também para a hemoglobina de mamíferos a constante de dissociação de $\mathrm{O}_{2}$ segue uma dependência de $\mathrm{pH}$ simples, a qual foi classicamente associada com o efeito Bohr alcalino. (ver Antonini e Brunori, 1971). Os parâmetros análogos para a hemoglobina humana são também apresentados na Tabela I.

\section{b) CINÉTICA DE COMBINAÇÃo DE MONóXIDO DE CARBONO}

Os experimentos de união de $\mathrm{CO}$ à hemoglobina de Loricarilchthys foram efetuados por mistura de: (i) uma solução contendo a proteína em tampão bis-tris ou tris a diferentes valores de $\mathrm{pH}$, na presença de $2,5 \mathrm{mM}$ de ATP e ditionito com (ii) uma solução contendo $8,5 \times 10^{-5} \mathrm{M}$ de $\mathrm{CO}$ em água sem gás. Fig. 2 mostra o curso de tempo da combinação de $\mathrm{CO}$ a dois valores de $\mathrm{pH}: 6,07$ e 8,28 . Estes experimentos foram efetuados sob condições de excesso do Jigante além da concentração de heme. Assim, os dados foram tratados como um processo de aparente $1^{\mathrm{a}}$ ordem. Pode-se notar, por comparação dos dois experimentos

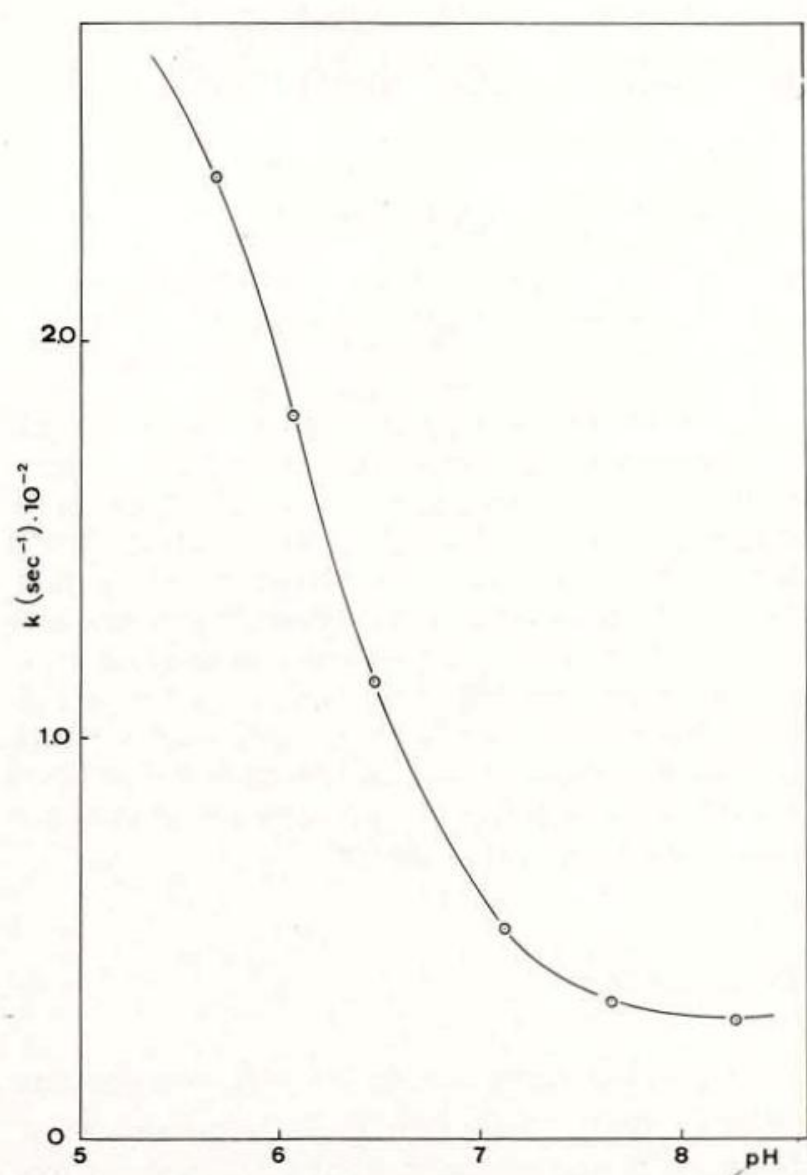

Fig. 1 - Dependência da constante de velocidade de $1 .{ }^{a}$ ordem para dissociação de $\mathrm{O}_{2} \mathrm{em} \mathrm{pH}$. A rea. ção foi medida pelo método do ditionito em tam. pões bis-tris ou tris, $0,2 \mathrm{M} \mathrm{C} 1-+1,25 \mathrm{mM}$ ATP. Temperatura $20^{\circ} \mathrm{C}$.

TABELA I - Cinética da dissociação de oxigênio de hemoglobina de Loricariichthys e humana.

\begin{tabular}{lrrrr}
\hline \multicolumn{1}{c}{ Espécies } & $\begin{array}{c}\text { K(ócl- } \\
\text { do)s-1 }\end{array}$ & $\begin{array}{c}\text { K(alca- } \\
\text { Uno)s-1 }\end{array}$ & pK app. & Temp. \\
\hline Loricariichthys sp. & 310 & 28 & 6.15 & 20 \\
Homem & 55 & 10 & 7.0 & 18 \\
\hline
\end{tabular}

na Fig. 2, que o curso de tempo de união do ligante é de aparente $1^{\mathrm{a}}$ ordem a pH 8,28 , indicando que os efeitos de interação hemeheme cineticamente observáveis não são evidentes a este $\mathrm{pH}$. Por outro lado, a valores inferiores de $\mathrm{pH}$ (por ex: $\mathrm{pH}$ 6,07), a combinação é mais lenta e claramente autocatalítica. 
Isto mostra que o sistema é cooperativo a baixos valores de pH. A Fig. 3 mostra a dependência de $\mathrm{pH}$ da velocidade média de combina-

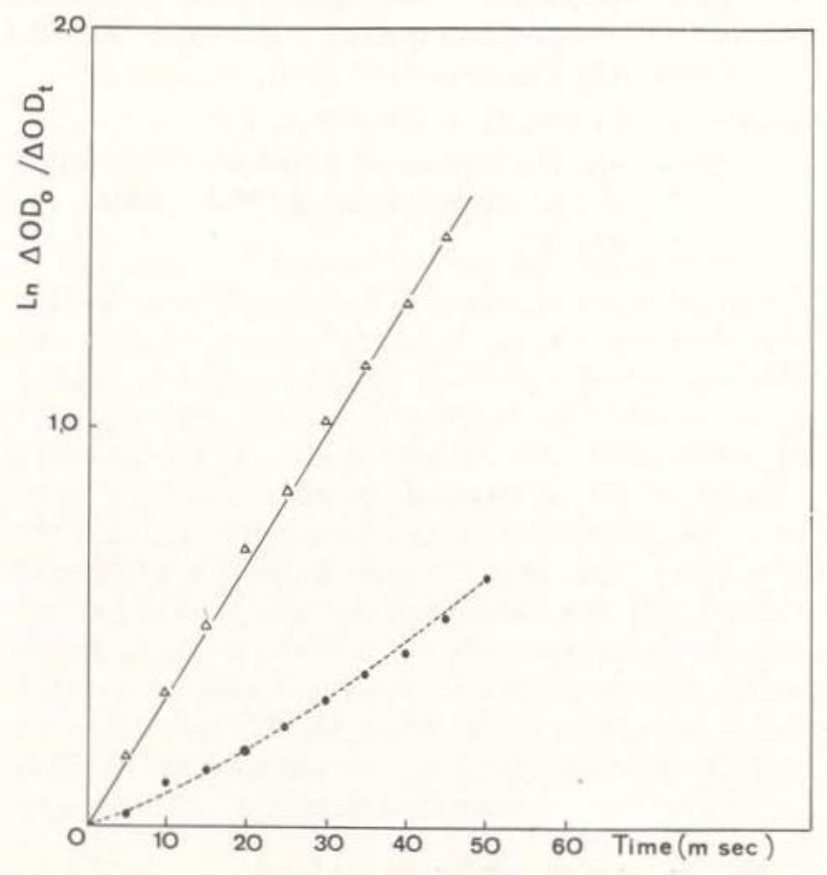

Fig. 2-Curso de tempo da combinação de CO com deoxiemoglobina a pH 6,07 (círculos) e 8,28 (triângulos). CO após mistura $=4.3 \times 10^{-5} \mathrm{M}$, ATP após mistura $=1,25 \mathrm{mM}, \mathrm{t}=20^{\circ} \mathrm{C}$, comprimento de onda de observação, $420 \mathrm{~nm}$.

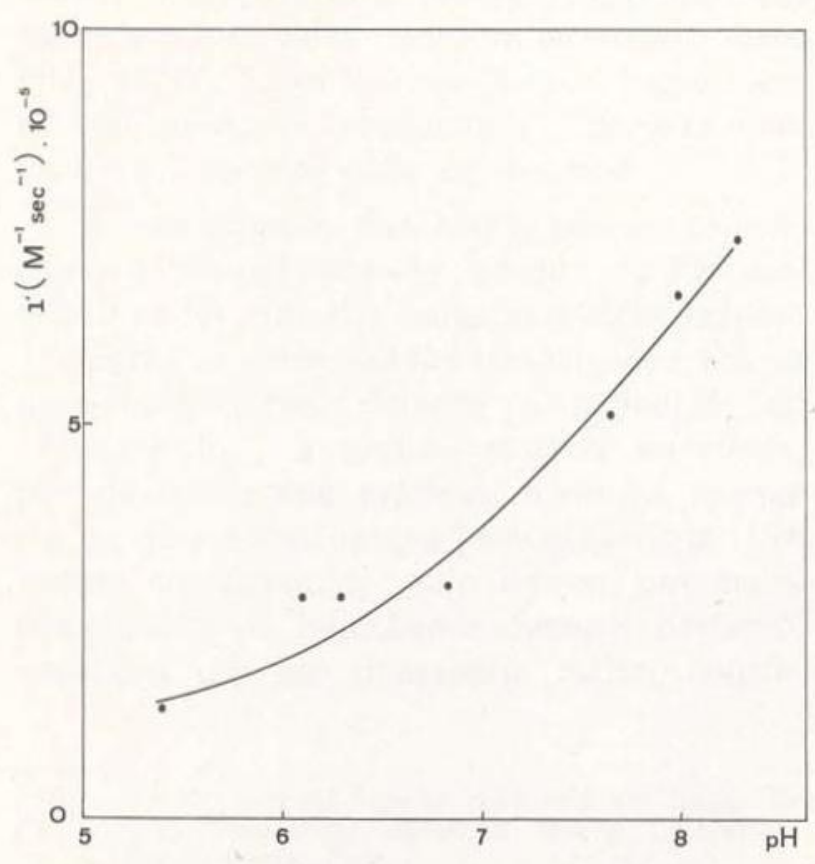

Fig. 3 - Dependência da constante de velocidade de 2.0 ordem para combinação de $\mathrm{CO}$ em pH. Condiçōes experimentais como na Fig. 1. ção total para CO (medida por volta de $50 \%$ de reação), e indica a presença de um significativo efeito Bohr normal. Se introduzirmos as conhecidas relações entre características de cooperatividade e afinidade de um modelo doisestágios (Monod et. al. 1965), estes resultados sugerem que a $\mathrm{pH}>7$ a hemoglobina de Loricariichthys é caracterizada por alta afinidade e baixa cooperatividade, enquanto a $\mathrm{pH}<7$, os efeitos cinéticos de cooperatividade tornamse evidentes e a afinidade total ligante decresce.

\section{AgRADECIMENTOS}

Este trabalho foi financiado pela National Science Foundation Grant PCM 75-06451. Somos gratos ao Governo Brasileiro pela permissão da entrada do "Alpha Helix" nas águas do rio Amazonas. M. Brunori, expressa seus agradecimentos ao National Research Council (CNR) da Itália pelo financiamento da viagem. M. T. agradece o suporte financeiro para a viagem concedido pela Royal Society, U. K. e Duke University, USA. A Focese Jr., recebeu auxílio parcial do Conselho Nacional de Ciência e Tecnologia. J.B. Bonaventura agradece a subvenção recebida de NIH Grant HL-15460 e de ONR Contract ND-0014-75-C-0190.

\section{SUMMARY}

Loricariichthys sp., an air-breathing fish from the Amazon River has one major hemoglobin component. Quantitative studies on the kinetics of $\mathrm{O}_{2}$ dissociation and $\mathrm{CO}$ combination to the protein were performed by stopped-flow experiments at different $\mathrm{pH}$ values and a constant ATP concentration of $1.25 \mathrm{mM}$. The oxygen dissociation shows a simple first order behavior and a strong $\mathrm{pH}$ dependence. The CO combination kinetics, on the other hand, were homogeneous and fast at higher $\mathrm{pH}$ values and slow and clearly autocatalytic at $\mathrm{pH}$ values below 7.0 .

\section{BIBLIOGRAFIA}

ANTONINI, E. \& BRUNORI, M.

1971 - Hemoglobin and Myoglobin in their Reactions with Ligands, North-Holland, Amsterdam. 
Brunori, M.; BONAVENTURA, J.; Focesi JR., A.; Galdames-Portus, M.I. \& Wilson, M.T. 1978 - Separação e caracterização dos componentes de hemoglobina de Pterygoplichthys pardalis, o acaribodo. Acta Ama zonica. $8(4)$ : Suplemento. (Este volume).

FINK, W.L. \& FINK, S.V.

1978 - A Amazônia Central e seus peixes. Acta Amazonica 8(4): Suplemento. (Este volume).

FYHN, U.E.H.; FYhN, H.J.; DAVIS, B.J.; POWERS, D.A.; FINK, W.L. \& GARLICK, R.L.

1978 - Heterogeneidade de hemoglobina nos peixes da Amazônia. Acta Amazonica 8(4): Suplemento. (Este volume).

GIBSON, Q.H. \& MILNES, L.

1964 - Apparatus for Rapid and sensitive spectrophotometry. Biochem. J., 91 : 162-171.

Monod, J. Wyman, J. \& Changeux, J.P.

1965 - On the Nature of Allosteric Transitions: A Plausible Model. J. Mol. Biol., 12: 88-118. 